\title{
A Singular Perturbation Approach for Time-Domain Assessment of Phase Margin
}

\author{
J. Jim Zhu, Xiaojing Yang and A. Scottedward Hodel
}

\begin{abstract}
This paper considers the problem of time-domain assessment of the Phase Margin (PM) of a Single Input Single Output (SISO) Linear Time-Invariant (LTI) system using a singular perturbation approach, where a SISO LTI fast loop system, whose phase lag increases monotonically with frequency, is introduced into the loop as a singular perturbation with a singular perturbation (time-scale separation) parameter $\varepsilon$. First, a bijective relationship between the Singular Perturbation Margin (SPM) $\varepsilon_{\max }$ and the PM of the nominal (slow) system is established with an approximation error on the order of $\varepsilon^{2}$. In proving this result, relationships between the singular perturbation parameter $\varepsilon$, PM of the perturbed system, PM and SPM of the nominal system, and the (monotonically increasing) phase of the fast system are also revealed. These results make it possible to assess the $P M$ of the nominal system in the time-domain for SISO LTI systems using the SPM with a standardized testing system called "PM-gauge," as demonstrated by examples. PM is a widely used stability margin for LTI control system design and certification. Unfortunately, it is not applicable to Linear Time-Varying (LTV) and Nonlinear Time-Varying (NLTV) systems. The approach developed here can be used to establish a theoretical as well as practical metric of stability margin for LTV and NLTV systems using a standardized SPM that is backward compatible with PM.
\end{abstract}

\section{INTRODUCTION}

Due to complexity of the dynamics of practical systems, feedback controllers are designed almost invariably based on simplified but concrete mathematical models known as nominal systems under some simplifying assumptions. However, the designed controllers will be implemented on the actual systems, which differ from the mathematical nominal model in many uncertain ways that are known as (structural) perturbations to the nominal system. The actual system or any mathematical model with higher fidelity than the nominal model are then called the perturbed system. In nonlinear control theory, perturbations that do not change the order of the nominal system, such as parametric dispersions or time-dependent variations, and negligible nonlinearities, are called regular perturbations; whereas those that change (increase) the order of the nominal system, such as fast parasitic dynamics that are ignored during controller design, are known as singular perturbations. An effective controller must be able to guarantee stability of the actual system

This work was supported in part by NASA Grant NNX07AN46G.

J. Jim Zhu is with Faculty of Electrical Engineering and Computer Science, Ohio University, Athens, OH 45701, USA zhuj@ohio.edu

Xiaojing Yang is with the Department of Electrical Engineering, Ohio University, Athens, OH 45701, USA xy140008@ohio.edu

Dedicated to A. Scottedward Hodel, who was with Faculty of Electrical and Computer Engineering Department, Auburn University before he passed away on January 9, 2009 during this collaborative work. in the presence of these perturbations. References [1]-[3] present an overview of singular perturbations including perturbation models, numerical and analytical treatments, ideas, results, and the applications of singular perturbation theory in different fields.

The extent to which a controller accommodate structural perturbations is called stability margin. Note that stability margin is different from the concept of domain of attraction, which is the ability of the controller to cope with perturbations in initial states. It is also different from the concept of input-output sensitivity (input-output gain), which indicates the ability of the closed-loop system to attenuates perturbations in the input, such as disturbances, infeasible tracking command trajectories or noises. Based upon the perturbation categories, stability margin can be divided into singular and regular perturbation margins. For Single-Input-Single-Output (SISO) Linear Time-Invariant (LTI) systems, Gain Margin (GM) and Phase Margin (PM) have been successfully used to gauge a nominal system's capability in accommodating parametric uncertainties (regular perturbation) and parasitic dynamics (singular perturbation). While parametric perturbations do affect the $\mathrm{PM}, \mathrm{PM}$ is predominantly a margin for accommodating parasitic dynamics as a singular perturbation margin. Whereas parasitic dynamics (with non-unity gain) do affect the loop gain, GM can be considered predominantly a regular perturbation margin. References [4]-[6] have discussed Nyquist robust stability margin, time-delay stability margin and so on, in the fields of robust control and $L_{1}$ adaptive control including regular and singular perturbation margins from the view of above classification.

However, since PM and GM are based on frequency response of LTI systems, they are not applicable to Linear Time-Varying (LTV), Non-Linear (NL) systems (including NLTI and NLTV) systems, which do not lend themselves to frequency domain analysis or synthesis. A common practice in controller design for LTV and NL systems applies the LTI controller design methods at discrete operating states within the operating envelope or along a nominal trajectory under the assumptions that the nonlinearity is benign and time-variance of the system parameters or nominal trajectory are slow. In operation, the controller gains are "scheduled" according to the current operating state or commanded trajectory. In essence, such gain-scheduled controllers and the closed-loop system are NLTV in nature. But the current practice is to certify such controllers by the 
GM and PM at the design points, which is well-known to be neither necessary nor sufficient for stability should the assumptions of benign nonlinearity and slowly-varying fail in operation, such as departure or upset, or simply flying a maneuvering trajectory such as in a flight control system, thereby jeopardizing the safety of the system. The benign nonlinearity and slowly-varying assumptions also limit the performance of the control system.

In recent years many advanced nonlinear and time-varying control techniques have been developed for NLTV systems to alleviate the benign nonlinearity and slowly-varying assumptions, thereby fully exploiting their performance potentials and improving their safety. However, there does not appear to have been reported for NLTV systems any stability margin metrics that are: (i) theoretically based, (ii) practically measurable, (iii) backward compatible in the sense that when applied to LTI systems, these metrics have a bijective correspondence with the GM and PM [7]. The lack of such effective stability metrics has inevitably hampered the acceptance of advanced LTV and NL control techniques in control engineering practices.

In this paper, we propose a time-domain approach for assessing PM of SISO LTI systems based on the singular perturbation theory, which can potentially be extended to LTV and NL systems as an effective gauge of stability margin for parasitic dynamics similar to what PM does for LTI systems.

The research on singularly perturbed systems with twoor multi-time scale are of theoretical and practical interests [8]-[10]. When the general form of the LTI autonomous singularly perturbed system is considered, it can be represented as [11]

$$
\begin{aligned}
\dot{x}(t) & =A_{11} x(t)+A_{12} z(t) \\
\varepsilon \dot{z}(t) & =A_{21} x(t)+A_{22} z(t)
\end{aligned}
$$

where $x(t) \in R^{n}, z(t) \in R^{m}$ are state vectors of slow and fast dynamics (two-time scale), respectively; $A_{11}, A_{12}, A_{21}$, $A_{22}$ are constant matrices and $A_{22}$ is Hurwitz; $\varepsilon$ is a small positive parameter that describes the time-scale separation, such as the ratio between the bandwidths or between the time-constants of the slow and fast dynamics. When $\varepsilon=0$, (1) reduces to the nominal system

$$
\dot{x}(t)=\left[A_{11}-A_{12} A_{22}^{-1} A_{21}\right] x(t)
$$

In [12] and [13], the perturbed system (1) was decomposed with Chang transformation [14] into two decoupled subsystems of the slow and fast dynamics. This result was generalized in [15] (see also [16]) to multi-time-scale singularly perturbed linear systems. The multi-time-scale decomposition can be used for implementing linear dynamic controllers, observers, and Kalman filters using different sampling rates.
Many results on stability of singularly perturbed NLTV systems are available (cf. Chapter 11 of [11] and Tikhonov's Theorem, p434 of [11]), which, roughly speaking, establish that when the null (quasi) equilibrium states of both the nominal operating error dynamics (defined by the slow system trajectory relative to the nominal operating state or trajectory) and the boundary layer dynamics (defined by the fast system trajectory relative to the slow dynamics) are exponentially stable, there exists a constant $\varepsilon_{\max }>0$ such that the null equilibrium of the singularly perturbed system (the composite system) is exponentially stable for all $\varepsilon<\varepsilon_{\max }$. We may call $\varepsilon_{\max }$ Singular Perturbation Margin (SPM). While both SPM and PM provide an indication of the stability margin of the nominal (slow) system for parasitic dynamics, the quantitative relationship between the SPM $\varepsilon_{\max }$ and the PM does not appear to have been studied.

The main results of this paper offer a singular perturbation approach for time-domain assessment of phase margin. Here, the nominal system (2) is assumed to have been (exponentially) stabilized with a healthy PM, and the fast system can be written as

$$
\begin{aligned}
\varepsilon \dot{z}(t) & =A_{22} z(t)+B_{\text {fast }} x(t) \\
y(t) & =C_{\text {fast }} z(t)
\end{aligned}
$$

where $C_{\text {fast }}=A_{12}$ and $B_{\text {fast }}=A_{21}$. Fast system (3) is assumed to be a monotonic-lag system (i.e. phase lag increases monotonically with frequency) with sufficiently large phase lag (larger than the PM of the nominal system) at the gain cutoff frequency $\omega_{\mathrm{cg}, \text { nom }}$ of the nominal system for sufficiently small $\varepsilon$. It will be shown that the PM of the perturbed system can be approximated by the sum of the PM of the nominal system and the phase of the fast system at $\omega_{\text {cg,nom }}$ of the nominal system with an error on the order of $\varepsilon^{2}$. With the $\omega_{\mathrm{cg}, \text { nom }}$ and $\varepsilon_{\max }$, our Theorem 1 establishes a bijective relationship between the SPM $\varepsilon_{\max }$ and the PM of the nominal system with an approximation error on the order of $\varepsilon^{2}$. This result can be used to assess the PM of the nominal system in the time domain when the SPM $\varepsilon_{\max }$ is found either by direct calculation of the eigenvalues of the perturbed system, or estimated using a singular perturbation approach. In practice, the fast system is designed as a "PM gauge," which should be optimized and standardized in order to obtain a quantitative estimate of PM of the nominal system. Proof of Theorem 1 revealed that the PM estimation error is due to the magnitude frequency response of the fast system at $\omega_{\mathrm{cg}, \text { nom }}$, therefore the optimal PM gauging system should be an allpass filter with unity gain for all $\varepsilon<\varepsilon_{\text {max }}$. The usage and effectiveness of this method are shown by examples.

The significance of the main results of this paper is not merely an alternative method for determining the PM of a SISO LTI system; rather it provides an avenue for defining a stability margin for LTV and NL systems that is theoretically 
based, practically measurable and backward compatible with PM. The extension relies on theoretically rigorous and practically computable algorithms for computing the SPM for LTV and NL systems. For LTV systems, a recently developed differential algebraic spectral theory [17]-[22] could be used to assess the SPM in a manner similar to the aforementioned Chang transformation and multi-time-scale decomposition for LTI systems. The LTV SPM methods, once developed, could be extended to NLTV systems by virtue of the equivalence of exponential stability of an equilibrium state of a NL system and its linearization thereof. For NL systems (including LTV systems as a special class), well-known Lyapunov function based singular perturbation results (see, for instance, Theorems 11.3 and 11.4 in [11]) can be used to obtain conservative estimates of SPM. In this latter approach, a recent result on spectral Lyapunov function [23] would prove to be useful.

The paper is organized as follows. After this introductory section, our main theoretical results will be presented in Section 2. The singular perturbation approach for time-domain assessment of PM is illustrated by examples in Section 3. Section 4 concludes the paper with some insightful remarks and suggestions for further research.

\section{MAIN RESULT}

The symbols used in the theorem statement and proof are shown in TABLE I.

TABLE I

SYMBOLS

\begin{tabular}{|c|c|c|}
\hline Symbols & Meanings & Unit \\
\hline$P M_{\mathrm{pt}}$ & Phase margin of the perturbed system & $\mathrm{rad}$ \\
\hline$P M_{\text {nom }}$ & Phase margin of the nominal system & $\mathrm{rad}$ \\
\hline$P M_{\text {fast }}$ & Phase margin of the fast system & $\mathrm{rad}$ \\
\hline$\phi_{\mathrm{pt}}(\omega, \varepsilon)$ & $\begin{array}{l}\text { Phase of the perturbed system at freq. } \omega \text {, } \\
\text { with singular perturbation parameter } \varepsilon\end{array}$ & $\mathrm{rad}$ \\
\hline$\phi_{\text {nom }}(\omega)$ & Phase of the nominal system at freq. $\omega$ & $\mathrm{rad}$ \\
\hline$\phi_{\text {fast }}(\omega, \varepsilon)$ & $\begin{array}{l}\text { Phase of the fast system at frequency } \omega \text {, } \\
\text { with singular perturbation parameter } \varepsilon\end{array}$ & $\mathrm{rad}$ \\
\hline$M_{\mathrm{pt}}(\omega, \varepsilon)$ & $\begin{array}{l}\text { Magnitude of the perturbed system at freq. } \\
\omega \text {, with singular perturbation parameter } \varepsilon\end{array}$ & $\mathrm{dB}$ \\
\hline$M_{\mathrm{nom}}(\omega)$ & Magnitude of nominal system at freq. $\omega$ & $\mathrm{dB}$ \\
\hline$M_{\text {fast }}(\omega, \varepsilon)$ & $\begin{array}{l}\text { Magnitude of the fast system at freq. } \omega \text {, } \\
\text { with singular perturbation parameter } \varepsilon\end{array}$ & $\mathrm{dB}$ \\
\hline$\omega_{\mathrm{cg}, \mathrm{pt}}$ & Perturbed system gain crossover frequency & $\mathrm{rad} / \mathrm{sec}$ \\
\hline$\omega_{\mathrm{cg}, \mathrm{nom}}$ & Nominal system gain crossover frequency & $\mathrm{rad} / \mathrm{sec}$ \\
\hline$\omega_{\mathrm{BW}, \mathrm{nom}}$ & Bandwidth of the nominal system & $\mathrm{rad} / \mathrm{sec}$ \\
\hline$\omega_{\mathrm{BW} \text {,fast }}$ & Bandwidth of the fast system & $\mathrm{rad} / \mathrm{sec}$ \\
\hline$L_{\mathrm{pt}}(s, \varepsilon)$ & $\begin{array}{l}\text { Perturbed system open loop transfer function } \\
\text { with singular perturbation parameter } \varepsilon\end{array}$ & \\
\hline$L_{\text {fast }}$ & $\begin{array}{l}\text { Fast system open loop transfer function, } \\
\text { with singular perturbation parameter } \varepsilon\end{array}$ & \\
\hline
\end{tabular}

The singular perturbation parameter $\varepsilon$ is defined by

$$
\varepsilon=\frac{\omega_{\mathrm{BW}, \text { nom }}}{\omega_{\mathrm{BW}, \text { fast }}} \approx \frac{\omega_{\mathrm{cg}, \text { nom }}}{\omega_{\mathrm{BW}, \text { fast }}}
$$

and $\varepsilon_{\max }$ is defined to the smallest $\omega_{\mathrm{BW} \text {,fast }}$ that makes the perturbed loop system be the $\varepsilon$ at onset of instability. The magnitude $M(\omega)$ of transfer function $L$ is defined by

$$
M(\omega)=20 \log |L(j \omega)|
$$

The perturbed system (1) is shown in Fig.1.

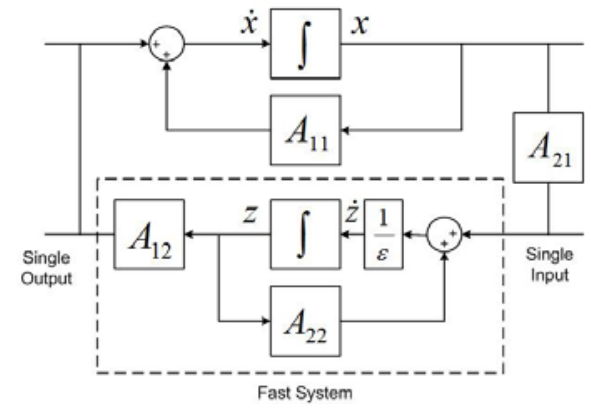

Fig. 1. Singular Perturbed System

The transfer function of the fast system (3) is given by

$$
L_{\text {fast }}(s, \varepsilon)=C_{\text {fast }}\left[\varepsilon s I-A_{22}\right]^{-1} B_{\text {fast }}
$$

\section{A. Theorem 1}

Consider the singularly perturbed LTI system (1), where $x(t) \in \mathbb{R}^{n}, z(t) \in \mathbb{R}^{m} ; A_{11}, A_{12}, A_{21}, A_{22}$ are constant matrices and $A_{22}$ is Hurwitz; $\varepsilon>0$ is the perturbation parameter. If the fast system is a SISO lag system with unity DC gain and monotonic phase lag that is sufficiently large for sufficiently small $\varepsilon$, then $P M_{\text {nom }}$ and $\varepsilon_{\max }$ satisfies a bijective relationship defined by

$$
P M_{\text {nom }}=\left|\phi_{\text {fast }}\left(\omega_{\text {cg,nom }}, \varepsilon_{\text {max }}\right)\right|+O\left(\varepsilon^{2}\right)
$$

Proof of Theorem 1 relies on the following lemma, which is an important result in its own rights.

\section{B. Lemma 1}

Consider the singular perturbed LTI system (1), with the same assumptions and definitions of the parameters in Theorem 1. The following relation between $P M_{\mathrm{pt}}, P M_{\mathrm{nom}}$ and $\phi_{\text {fast }}$ is satisfied

$$
P M_{\mathrm{pt}}=P M_{\mathrm{nom}}+\phi_{\mathrm{fast}}\left(\omega_{\mathrm{cg}, \mathrm{nom}}, \varepsilon\right)+O\left(\varepsilon^{2}\right)
$$

\section{Proof of Lemma 1}

Suppose $A_{22}$ is Hurwitz, the loop transfer function of the fast system, which is a rational function with unity DC gain, and poles having negative real parts, can be written as

$L_{\text {fast }}(s)=\frac{\prod_{i=1}^{M_{1}}\left[\varepsilon s / z_{i}+1\right] \prod_{i=1}^{M_{2}}\left[\left(\varepsilon s / \omega_{m_{i}}\right)^{2}+\left(2 \zeta_{m_{i}} / \omega_{m_{i}}\right) \varepsilon s+1\right]}{\prod_{j=1}^{N_{1}}\left[\varepsilon s / p_{j}+1\right] \prod_{j=1}^{N_{2}}\left[\left(\varepsilon s / \omega_{n_{j}}\right)^{2}+\left(2 \zeta_{n_{j}} / \omega_{n_{j}}\right) \varepsilon s+1\right]}$

The fast system is a phase lag system, so

$$
\min \left\{\left\{z_{i}\right\}_{i=1}^{M_{1}} \cup\left\{\omega_{m_{i}}\right\}_{i=1}^{M_{2}}\right\}>\min \left\{\left\{p_{j}\right\}_{j=1}^{N_{1}} \cup\left\{\omega_{n_{j}}\right\}_{j=1}^{N_{2}}\right\}
$$


and

$$
\begin{aligned}
& \left|\prod_{i=1}^{M_{1}}\left[\varepsilon s / z_{i}+1\right] \prod_{i=1}^{M_{2}}\left[\left(\varepsilon s / \omega_{m_{i}}\right)^{2}+\left(2 \zeta_{m_{i}} / \omega_{m_{i}}\right) \varepsilon s+1\right]\right| \\
< & \left|\prod_{j=1}^{N_{1}}\left[\varepsilon s / p_{j}+1\right] \prod_{j=1}^{N_{2}}\left[\left(\varepsilon s / \omega_{n_{j}}\right)^{2}+\left(2 \zeta_{n_{j}} / \omega_{n_{j}}\right) \varepsilon s+1\right]\right|
\end{aligned}
$$

Let

$$
\omega_{\min }=\min \left\{\left\{p_{j}\right\}_{j=1}^{N_{1}} \cup\left\{\omega_{n_{j}}\right\}_{j=1}^{N_{2}}\right\}
$$

and for the magnitude of $L_{\text {fast }}$, we have

$$
\begin{aligned}
< & N_{1}|20 \log | \frac{1}{\varepsilon j \frac{\omega_{\text {cg,nom }}}{\omega_{\min }}+1}|| \\
& +N_{2}|20 \log | \frac{1}{1+\varepsilon j \frac{2 \zeta \omega_{\text {cg,nom }}}{\omega_{\min }}+\left(\varepsilon j \frac{\omega_{\text {cg,nom }}}{\omega_{\min }}\right)^{2}}||
\end{aligned}
$$

where $\zeta \in(0,1)$. By Taylor Expansion of (9) at the point $\varepsilon=0$, we obtain

$$
\begin{aligned}
& \left|M_{\text {fast }}\left(\omega_{\text {cg,nom }}\right)\right| \\
< & 20 N_{1}\left|\frac{-\omega_{\text {cg,nom }}^{2}}{2(\ln 10) \omega_{\min }^{2}} \varepsilon^{2}+O\left(\varepsilon^{4}\right)\right| \\
& +20 N_{2}\left|\frac{\left(1-2 \zeta^{2}\right) \omega_{\mathrm{cg}, \text { nom }}^{2}}{(\ln 10) \omega_{\min }^{2}} \varepsilon^{2}+O\left(\varepsilon^{4}\right)\right| \\
= & O\left(\varepsilon^{2}\right)
\end{aligned}
$$

Since $M_{\text {nom }}\left(\omega_{\text {cg,nom }}\right)=0$, the magnitude of the perturbed system at $\omega_{\text {cg,nom }}$ is

$$
\begin{aligned}
M_{\mathrm{pt}}\left(\omega_{\mathrm{cg}, \mathrm{nom}}\right) & =M_{\text {nom }}\left(\omega_{\mathrm{cg}, \mathrm{nom}}\right)+M_{\text {fast }}\left(\omega_{\mathrm{cg}, \mathrm{nom}}\right) \\
& =M_{\text {fast }}\left(\omega_{\mathrm{cg}, \mathrm{nom}}\right) \\
& =O\left(\varepsilon^{2}\right)
\end{aligned}
$$

which is shown by Fig.2.

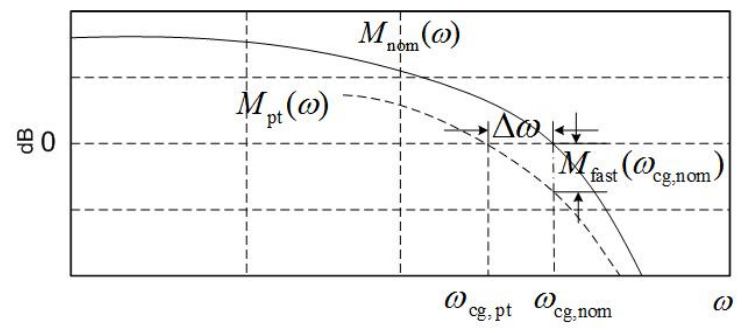

Fig. 2. Magnitude-Frequency Plot

Let $|\Delta \omega|=\left|\omega_{\mathrm{cg}, \mathrm{pt}}-\omega_{\mathrm{cg}, \mathrm{nom}}\right|$, and by the mean value theorem, we have

$$
\frac{\left|M_{\mathrm{pt}}\left(\omega_{\mathrm{cg}, \text { nom }}\right)\right|}{|\Delta \omega|}=\left|\frac{\mathrm{d}}{\mathrm{d} \omega} M_{\mathrm{pt}}\left(\omega_{0}\right)\right|
$$

for some $\omega_{0} \in\left[\min \left\{\omega_{\mathrm{cg}, \mathrm{pt}}, \omega_{\mathrm{cg}, \mathrm{nom}}\right\}, \max \left\{\omega_{\mathrm{cg}, \mathrm{pt}}, \omega_{\mathrm{cg}, \mathrm{nom}}\right\}\right]$. It then follows from (12) and the last equality in (11) that

$$
|\Delta \omega|=\frac{\left|M_{\text {fast }}\left(\omega_{\mathrm{cg}, \mathrm{nom}}\right)\right|}{\left|\frac{\mathrm{d}}{\mathrm{d} \omega} M_{\mathrm{pt}}\left(\omega_{0}\right)\right|}=O\left(\varepsilon^{2}\right)
$$

Let $\widetilde{P M}_{\mathrm{pt}}=P M_{\mathrm{nom}}+\phi_{\mathrm{fast}}\left(\omega_{\mathrm{cg}, \text { nom }}\right)$, and

$$
\begin{aligned}
& \left|P M_{\mathrm{pt}}-\widetilde{P M}_{\mathrm{pt}}\right| \\
& =\mid\left(\pi+\phi_{\text {nom }}\left(\omega_{\text {cg,pt }}\right)+\phi_{\text {fast }}\left(\omega_{\text {cg,nom }}\right)\right) \\
& -\left(P M_{\text {nom }}+\phi_{\text {fast }}\left(\omega_{\mathrm{cg}, \text { nom }}\right)\right) \mid \\
& =\mid\left(\pi+\phi_{\text {nom }}\left(\omega_{\text {cg,pt }}\right)+\phi_{\text {fast }}\left(\omega_{\text {cg,nom }}\right)\right) \\
& -\left(\pi+\phi_{\text {nom }}\left(\omega_{\text {cg,nom }}\right)+\phi_{\text {fast }}\left(\omega_{\text {cg,nom }}\right)\right) \mid \\
& \leq\left|\phi_{\text {nom }}\left(\omega_{\text {cg,pt }}\right)-\phi_{\text {nom }}\left(\omega_{\text {cg,nom }}\right)\right| \\
& +\left|\phi_{\text {fast }}\left(\omega_{\text {cg,pt }}\right)-\phi_{\text {fast }}\left(\omega_{\text {cg,nom }}\right)\right| \\
& =|\Delta \omega|\left(\left|\frac{\mathrm{d}}{\mathrm{d} \omega} \phi_{\text {nom }}\left(\omega_{1}\right)\right|+\left|\frac{\mathrm{d}}{\mathrm{d} \omega} \phi_{\text {fast }}\left(\omega_{2}\right)\right|\right)
\end{aligned}
$$

for some $\omega_{1}, \omega_{2} \in\left[\min \left\{\omega_{\mathrm{cg}, \mathrm{pt}}, \omega_{\mathrm{cg}, \mathrm{nom}}\right\}, \max \left\{\omega_{\mathrm{cg}, \mathrm{pt}}, \omega_{\mathrm{cg}, \mathrm{nom}}\right\}\right]$, and then

$$
\left|P M_{\mathrm{pt}}-\widetilde{P M}_{\mathrm{pt}}\right|=O(|\Delta \omega|)=O\left(\varepsilon^{2}\right)
$$

Therefore

$$
P M_{\mathrm{pt}}=P M_{\mathrm{nom}}+\phi_{\mathrm{fast}}\left(\omega_{\mathrm{cg}, \mathrm{nom}}\right)+O\left(\varepsilon^{2}\right)
$$

\section{Proof of Theorem 1}

By assumption, the fast system has a monotonically increasing phase lag. When $P M_{\text {nom }}$ is given, $\left|\phi_{\text {fast }}\left(\omega_{\mathrm{cg}, \text { nom }}, \varepsilon\right)\right|$ increases as $\varepsilon$ increases, and $P M_{\mathrm{pt}}$ decreases. When $P M_{\mathrm{pt}}$ reaches 0 , we obtain $\varepsilon_{\max }$. By Lemma 1

$$
P M_{\mathrm{pt}}=0=P M_{\mathrm{nom}}+\phi_{\mathrm{fast}}\left(\omega_{\mathrm{cg}, \mathrm{nom}}, \varepsilon_{\mathrm{max}}\right)+O\left(\varepsilon^{2}\right)
$$

Therefore

$$
P M_{\text {nom }}=\left|\phi_{\text {fast }}\left(\omega_{\text {cg,nom }}, \varepsilon_{\text {max }}\right)\right|+O\left(\varepsilon^{2}\right)
$$

Notice that $\phi_{\text {fast }}(s, \varepsilon)=\phi_{\text {fast }}(s \varepsilon)$. It follows the assumption that $\phi_{\text {fast }}(s, \varepsilon)$ is a monotonically increasing function of $\omega$ that $P M_{\text {nom }}$ is a bijective function of the SPM $\varepsilon_{\max }$.

\section{Time-Domain Phase Margin Assessment}

In this section we will apply Theorem 1 and Lemma 1 to time-domain assessment of $P M_{\text {nom }}$ for the nominal system. We will assume the gain-crossover frequency $\omega_{\text {cg,nom }}$ is known for the given nominal system, which will be used to design a LTI singular perturbation (fast) system $L_{\text {fast }}(s, \mathcal{\varepsilon})$, called a PM-gauge, whose phase $\phi_{\text {fast }}\left(j \omega_{\mathrm{cg}, \mathrm{nom}}, \varepsilon_{\max }\right)$ will be used to determine $P M_{\text {nom }}$ according to Theorem 1, where the SPM $\varepsilon_{\max }$ can be determined in the frequency domain from the condition $P M_{\mathrm{pt}}\left(\varepsilon_{\mathrm{max}}\right)=0$. However, the purpose here is to calculate $\varepsilon_{\max }$ in the time-domain from the state equations of the perturbed system comprising of the nominal and the PM-gauge systems. In Subsection 3.1 we will first present both analytical and numerical methods for evaluating the SPM $\varepsilon_{\max }$ in the time-domain. Then in subsequent subsections we will present three types of PM-gauge, each of which has unique advantages and 
disadvantages that are suited for a certain applications.

The design methods and the effectiveness of the designed PM-gauges will be demonstrated using the nominal system shown in Fig.3 with a loop transfer function given by

$$
L_{\text {nom }}(s)=\frac{C_{\mathrm{P}} B_{\mathrm{P}}\left(K_{\mathrm{I}}+K_{\mathrm{P}} s\right)}{s\left(s+A_{\mathrm{P}}\right)}
$$

which is a first-order LTI plant with a Proportional-Integral (PI) feedback controller, where the plant parameters are given by $A_{\mathrm{P}}=1, B_{\mathrm{P}}=1, C_{\mathrm{P}}=1$. In order to illustrate the $O\left(\varepsilon^{2}\right)$ accuracy of estimated $P M_{\text {nom }}$ using different PM-gauges, two sets of controller gains $K_{\mathrm{I}}$ and $K_{\mathrm{P}}$ will be used, which result in two sets of $P M_{\text {nom }}$ and $\omega_{\text {cg,nom }}$ values, as shown in TABLE II.

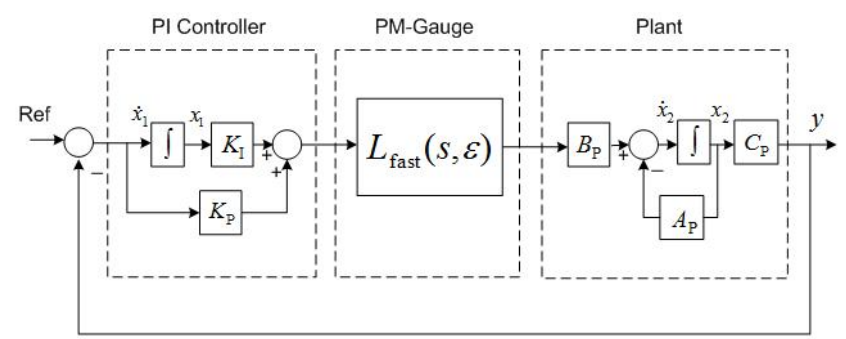

Fig. 3. The Nominal System

TABLE II

NOMINAL SySTEM PARAMETERS

\begin{tabular}{|c||c||c||c||c|}
\hline Parameters & $K_{\mathrm{P}}$ & $K_{\mathrm{I}}$ & $P M_{\text {nom }}$ & $\omega_{\text {cg,nom }}$ \\
\hline Case 1 & 1 & 3 & $\pi / 3$ & 1.7321 \\
\hline Case 2 & 0.5 & 7.83 & $\pi / 6$ & 2.7320 \\
\hline
\end{tabular}

\section{A. Time-Domain Calculation of SPM}

Consider the singularly perturbed LTI system (1) where $A_{22}$ is Hurwitz, and assume the nominal system (2) has been exponentially stabilized, i.e. $\left[A_{11}-A_{12} A_{22}^{-1} A_{21}\right]$ is Hurwitz. Then standard singular perturbation theory provides exponential stability of the perturbed system for sufficiently small $\varepsilon>0$. Thus, SPM $\varepsilon_{\max }$ can be defined as the least upper bound on $\varepsilon>0$ for which the perturbed system remains exponentially stable. The SPM $\varepsilon_{\max }$ can then be found by monotonically increasing $\varepsilon$ from a very small value until one of the eigenvalues of the perturbed system becomes imaginary (including zero).

To this end, first rewrite the perturbed system (1) as a composite system

$$
\left[\begin{array}{l}
\dot{x}(t) \\
\dot{z}(t)
\end{array}\right]=\left[\begin{array}{ll}
A_{11} & A_{12} \\
\frac{1}{\varepsilon} A_{21} & \frac{1}{\varepsilon} A_{22}
\end{array}\right]\left[\begin{array}{l}
x(t) \\
z(t)
\end{array}\right]=A(\varepsilon)\left[\begin{array}{l}
x(t) \\
z(t)
\end{array}\right]
$$

The characteristic equation of (17) can be written as $\operatorname{det}[\lambda I-A(\varepsilon)]=\lambda^{N}+a_{N}(\varepsilon) \lambda^{N-1}+\ldots+a_{2}(\varepsilon) \lambda+a_{1}(\varepsilon)=0$ where $N=n+m$. For $\varepsilon=\varepsilon_{\max }$, (18) has an imaginary eigenvalue $\lambda=j \beta$, which simultaneously satisfies the realpart and imaginary-part of (18)

$$
\begin{aligned}
(-1)^{k} a_{2 k+1} \beta^{2 k}+\ldots+a_{5}(\varepsilon) \beta^{4}-a_{3}(\varepsilon) \beta^{2}+a_{1}(\varepsilon) & =0 \\
(-1)^{k} a_{2(k+1)} \beta^{2 k}+\ldots+a_{6}(\varepsilon) \beta^{4}-a_{4}(\varepsilon) \beta^{2}+a_{2}(\varepsilon) & =0
\end{aligned}
$$

where $k=1,2, \ldots, M$ and $M=[N / 2]$, and $a_{N+1}(\varepsilon)=1$, $a_{N+2}(\varepsilon)=0$ when appropriate. Thus, a standard numerical method can be used to solve for $\beta$ from both equations in (19) for a given $\varepsilon$, and $\varepsilon=\varepsilon_{\max }$ is determined by increment $\varepsilon$ until the values for $\beta$ from both equations are sufficiently close. To speed up the search process, increment of $\varepsilon$ can be variable based on the difference between the two solutions for $\beta$ from both equations in (19).

When the order $N \leq 9$, it is possible to solve for $\varepsilon_{\max }$ from (19) analytically using the root formulas for quadratic, cubic and quartic polynomials, as illustrated below in the PM-gauge design examples.

\section{B. Minimum-Phase PM-Gauges}

Because minimum-phase zeros introduce monotonic phase leads, which violate the monotonic phase lag assumption for Theorem 1, a minimum-phase PM-gauge must be an all-pole system. Since the magnitude function of an all-pole system is not a constant, a minimum-phase PM-gauge will have an $O\left(\varepsilon^{2}\right)$ error in estimating $P M_{\text {nom }}$ according to Theorem 1. The following example illustrates a minimum-phase PM-gauge.

Consider a second-order minimum-phase PM-gauge with a transfer function

$$
L_{\mathrm{fast}}(s, \varepsilon)=\frac{\omega_{0}^{2}}{(\varepsilon s)^{2}+2 \zeta \omega_{0}(\varepsilon s)+\omega_{0}^{2}}
$$

The phase function of $L_{\mathrm{fast}}(j \omega, \varepsilon)$ is given by

$$
\left|\phi_{\text {fast }}(j \omega, \varepsilon)\right|=\arctan \left(\frac{2 \zeta \frac{\varepsilon \omega}{\omega_{0}}}{1-\left(\frac{\varepsilon \omega}{\omega_{0}}\right)^{2}}\right)
$$

We choose $\omega_{0}=\omega_{\mathrm{cg}, \mathrm{nom}}$, so that $\varepsilon_{\max }=1$ corresponds to $P M_{\text {nom }}=\pi / 2 \operatorname{rad}\left(90^{\circ}\right)$. By choosing $\zeta=1$ we have

$$
P M_{\mathrm{nom}} \simeq\left|\phi_{\mathrm{fast}}\left(j \omega_{\mathrm{cg}, \mathrm{nom}}, \varepsilon_{\mathrm{max}}\right)\right|=\arctan \left(\frac{2 \varepsilon_{\max }}{1-\varepsilon_{\max }^{2}}\right)
$$

with an $O\left(\varepsilon^{2}\right)$ error. The approximate relationship between PM and SPM is plotted in Fig.4. 


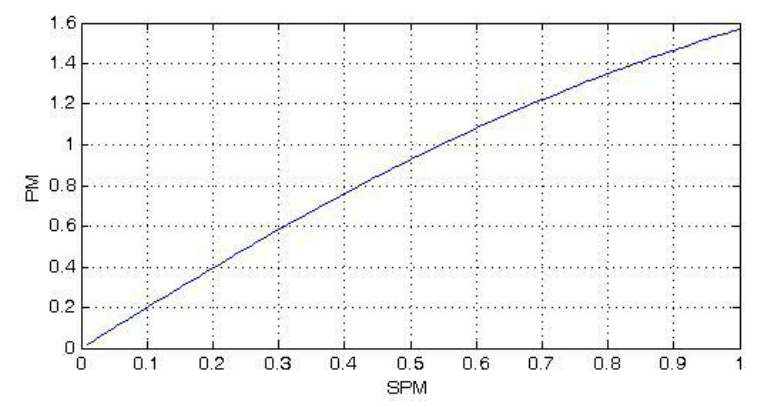

Fig. 4. Approximate Relationship between PM and SPM for (20)

For the two test cases of nominal systems specified in TABLE II, the transfer function of the corresponding PMgauges along with the corresponding SPM $\varepsilon_{\max }$ values, estimated $P M_{\text {nom }}$ values and the estimation errors, and the bound $k=\left(P M_{\text {nom }}\right.$ Error $) / \varepsilon_{\max }^{2}$ are shown in TABLE 2. The $O\left(\varepsilon^{2}\right)$ accuracy of the estimation error is clearly demonstrated. It is reassuring to know that the estimation error is significantly smaller as the design becomes more critical. The estimation error can be further reduced by using a higher order PMgauge, such as cascading two PM-gauges of (20). However, it will only reduce the constant $k$ in the last column of TABLE III, not by orders-of-magnitude.

TABLE III

Minimum-Phase PM-Gauge Test Results

\begin{tabular}{|c||c||c|}
\hline Parameters & $L_{\text {fast }}(s, \varepsilon)$ & SPM $\varepsilon_{\max }$ \\
\hline Case 1 & $\frac{3.0002}{(\varepsilon s)^{2}+3.4642(\varepsilon s)+3.0002}$ & 0.7087 \\
\hline Case 2 & $\frac{7.4638}{(\varepsilon s)^{2}+5.4640(\varepsilon s)+7.4638}$ & 0.2819 \\
\hline
\end{tabular}

\begin{tabular}{|l||c||c||c||c|}
\hline Param. & Est. $P M_{\text {nom }}$ & Exact $P M_{\text {nom }}$ & $P M_{\text {nom }}$ Err. & $\mathrm{k}$ \\
\hline Case 1 & 1.2331 & $\pi / 3=1.0472$ & $17.75 \%$ & 0.3701 \\
\hline Case 2 & 0.5495 & $\pi / 6=0.5236$ & $4.95 \%$ & 0.3259 \\
\hline
\end{tabular}

It is noted that the SPM $\varepsilon_{\max }$ determined in the frequency domain from $P M_{\mathrm{pt}}\left(\varepsilon_{\mathrm{max}}\right)$ and that determined using the time-domain method introduced above are identical. Since the order of the perturbed system is $N=4, \varepsilon_{\max }$ can be determined analytically using the quadratic root formula from (19), which now becomes

$$
\begin{aligned}
\beta^{4}-a_{3}(\varepsilon) \beta^{2}+a_{1}(\varepsilon) & =0 \\
a_{4}(\varepsilon) \beta^{2}+a_{2}(\varepsilon) & =0
\end{aligned}
$$

Eliminating $\beta^{2}$ from these equations yields an equation for $\varepsilon$

$$
a_{2}^{2}(\varepsilon)+a_{4}(\varepsilon) a_{3}(\varepsilon) a_{2}(\varepsilon)+a_{4}^{2}(\varepsilon) a_{1}(\varepsilon)=0
$$

which turns out to be a second-order polynomial in $\varepsilon^{2}$, and therefore can be solved using the quadratic root formula. Among the four roots, the smallest positive root is $\varepsilon_{\max }$.

\section{Zero-Error PM-Gauges}

From the proofs of Theorem 1 and Lemma 1 it is seen that the $O\left(\varepsilon^{2}\right)$ estimation error is due to the non-unity magnitude function of the PM-gauge $M_{\text {fast }}(s, \varepsilon)$. Thus it is possible to design zero-error PM-gauges using all-pass filter transfer functions $L_{\text {fast }}(s, \varepsilon)$ whose magnitude function $M_{\text {fast }}(s, \varepsilon)=1$. To illustrate the design, consider first-order all-pass filter transfer function

$$
L_{\text {fast }}(s, \varepsilon)=\frac{1-\frac{\varepsilon s}{\omega_{0}}}{1+\frac{\varepsilon s}{\omega_{0}}}
$$

whose phase function is given by

$$
\left|\phi_{\text {fast }}(j \omega, \varepsilon)\right|=\arctan \left(\frac{2 \frac{\varepsilon \omega}{\omega_{0}}}{1-\left(\frac{\varepsilon \omega}{\omega_{0}}\right)^{2}}\right)
$$

By choosing $\omega_{0}=\omega_{\text {cg,nom }}$, so that $\varepsilon_{\max }=1$ corresponds to $P M_{\text {nom }}=\pi / 2 \operatorname{rad}\left(90^{\circ}\right)$, we have

$$
P M_{\text {nom }}=\left|\phi_{\text {fast }}\left(j \omega_{\mathrm{cg}, \text { nom }}, \varepsilon_{\max }\right)\right|=\arctan \left(\frac{2 \varepsilon_{\max }}{1-\varepsilon_{\max }^{2}}\right)
$$

which is identical to (22). However, there is no estimation error, and the order of the PM-gauge is only half of that of the minimum-phase counterpart (20). The drawback of such zero-error PM-gauges is that they are necessarily nonminimum-phase, which may cause problem in simulation verifications of PM or SPM using such PM-gauges. The results of applying the zero-error PM-gauge (24) to the two test cases are documented in TABLE IV.

TABLE IV

Zero-Error PM-Gauge Test Results

\begin{tabular}{|c||c||c|}
\hline Parameters & $L_{\text {fast }}(s, \varepsilon)$ & SPM $\varepsilon_{\max }$ \\
\hline Case 1 & $\frac{1-\frac{\varepsilon s}{1.7321}}{1+\frac{\varepsilon s}{1.7321}}$ & 0.5774 \\
\hline Case 2 & $\frac{1-\frac{\varepsilon s}{2.7320}}{1+\frac{\varepsilon s}{2.7320}}$ & 0.2679 \\
\hline
\end{tabular}

\begin{tabular}{|c||c||c||c|}
\hline Parameters & Est. $P M_{\text {nom }}$ & Exact $P M_{\text {nom }}$ & $P M_{\text {nom }}$ Err. \\
\hline Case 1 & 1.0472 & $\pi / 3=1.0472$ & 0 \\
\hline Case 2 & 0.5236 & $\pi / 6=0.5236$ & 0 \\
\hline
\end{tabular}

\section{Approximately-Linear-Phase PM-Gauges}

Although the zero-error PM-gauge of the preceding section gives exact $P M_{\text {nom }}$ from SPM $\varepsilon_{\max }$, the relationship between them is a nonlinear function. In practice, it is desirable to have a linear relation between $P M_{\text {nom }}$ and SPM $\varepsilon_{\text {max }}$ with zero-error. A pure transport delay possesses the desired unity magnitude function and linear phase function. Thus, we may design linear-phase, zero-error PM-gauge as

$$
L_{\text {fast }}(s, \varepsilon)=e^{-T_{0} \varepsilon s}
$$

whose phase function is given by

$$
\left|\phi_{\text {fast }}(j \omega, \varepsilon)\right|=T_{0} \varepsilon s
$$

By choosing $T_{0}=\pi / \omega_{\mathrm{cg}, \text { nom }}$, so that $\varepsilon_{\mathrm{max}}=1$ corresponds to $P M_{\mathrm{nom}}=\pi \operatorname{rad}\left(180^{\circ}\right)$, we have

$$
P M_{\text {nom }}=\left|\phi_{\text {fast }}\left(j \omega_{\text {cg,nom }}, \varepsilon_{\text {max }}\right)\right|=\varepsilon_{\max } \pi
$$

While this is highly desirable, it is difficult to analyze the stability of the delay-perturbed system. This problem can 
be alleviated by using the Pade approximation to the delay transfer function, thereby obtaining approximately-linearphase PM-gauges. In fact, the zero-error PM-gauge in the preceding section is the first-order Pade approximation to the delay function, but its phase function is quite nonlinear. Now, consider the third-order Pade approximation to (27)

$$
L_{\mathrm{fast}}(s, \varepsilon)=\frac{120-60 T_{0} \varepsilon s+12\left(T_{0} \varepsilon s\right)^{2}-\left(T_{0} \varepsilon s\right)^{3}}{120+60 T_{0} \varepsilon s+12\left(T_{0} \varepsilon s\right)^{2}+\left(T_{0} \varepsilon s\right)^{3}}
$$

With $T_{0}=\pi / \omega_{\mathrm{cg}, \text { nom }}$ and by Taylor expansion at $\varepsilon_{\max }=0$, we have

$$
\begin{aligned}
P M_{\text {nom }} & =\left|\phi_{\text {fast }}\left(j \omega_{\mathrm{cg}, \text { nom }}, \varepsilon_{\max }\right)\right| \\
& =\left|2 \arctan \left(\frac{\left(\varepsilon_{\max } \pi\right)^{3}-60\left(\varepsilon_{\max } \pi\right)}{120-12\left(\varepsilon_{\max } \pi\right)^{2}}\right)\right| \\
& \simeq \varepsilon_{\max } \pi
\end{aligned}
$$

Note that the estimation error of $P M_{\text {nom }}$ using the exact phase function $\left|\phi_{\text {fast }}\left(j \omega_{\text {cg,nom }}, \varepsilon_{\max }\right)\right|$ is zero. The error of the linear approximation $P M_{\text {nom }} \simeq \varepsilon_{\max } \pi$ is given by

$\operatorname{err}\left(\varepsilon_{\max }\right)=\left|\frac{\varepsilon_{\max } \pi}{\left|2 \arctan \left(\frac{\left(\varepsilon_{\max } \pi\right)^{3}-60\left(\varepsilon_{\max } \pi\right)}{120-12\left(\varepsilon_{\max } \pi\right)^{2}}\right)\right|}-1\right| \times 100 \%$

which is a monotonically increasing function of $\varepsilon_{\max }$ maximizing at $\varepsilon_{\max }=1$ to $0.64 \%$, as shown in Fig.5.
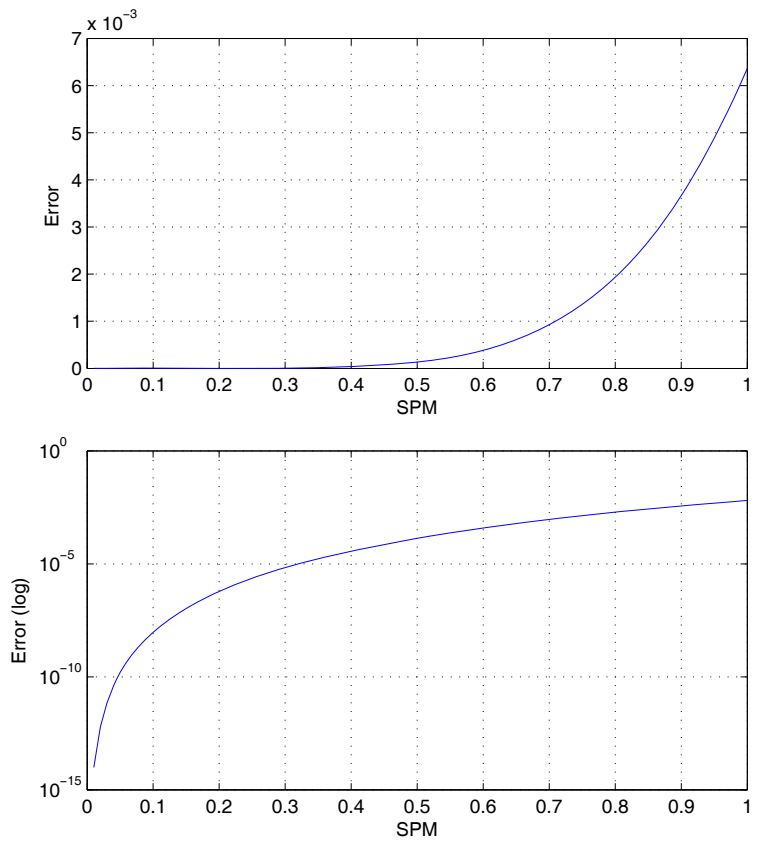

Fig. 5. Approximation Error for the 3rd Order PM-gauge (30)

Since practically $P M_{\text {nom }}$ is seldom more than $\frac{1}{3} \pi \mathrm{rad}$ $\left(60^{\circ}\right)$, for which the estimation error is given by

$$
\operatorname{err}\left(\varepsilon_{\max }\right) \leq \operatorname{err}\left(\frac{1}{3}\right)=0.00125 \%
$$

which is accurate enough for any practical purposes. The test results of the approximately-linear-phase PM-gauge on the two nominal systems are presented in TABLE V. The approximation errors shown in the TABLE $\mathrm{V}$ are due to two sources: the approximately linear phase and the finite precision in implementing the transfer function (30), where by (32) and (33). The estimation error by the linear approximation is indiscernible, and most of the errors are due to the precision of the implementation. The SPM $\varepsilon_{\max }$ values

\begin{tabular}{|c|c|c|c|c|}
\hline Parameters & \multicolumn{4}{|c|}{$L_{\text {fast }}(s, \varepsilon)$} \\
\hline Case 1 & \multicolumn{4}{|c|}{$\frac{120-108.8249 \varepsilon s+12(1.8137 \varepsilon s)^{2}-(1.8137 \varepsilon s)^{3}}{120+108.8249 \varepsilon s+12(1.8137 \varepsilon s)^{2}+(1.8137 \varepsilon s)^{3}}$} \\
\hline Case 2 & \multicolumn{4}{|c|}{$120-68.9954 T_{0} \varepsilon s+12(1.1499 \varepsilon s)^{2}-(1.1499 \varepsilon s)^{3}$} \\
\hline & $\varepsilon_{\mathrm{m}}$ & $\varepsilon_{\mathrm{n}}$ & Ex & $\varepsilon_{n}$ \\
\hline & 304 & 1.0380 & $\pi / 3=1.04$ & $0.8785 \%$ \\
\hline Case 2 & 0.1660 & 0.5216 & $\pi / 6=0.5236$ & $0.3909 \%$ \\
\hline
\end{tabular}
were obtained by solving (19) using the numerical method presented above using MATLAB.

TABLE V

Approximately-Linear-Phase PM-Gauge Test Results

The third-order approximately-linear-phase PM-gauge (30) does increase the difficulty in evaluating the SPM $\varepsilon_{\max }$ comparing to the previous two PM-gauge designs, but it is insignificant, and in fact worthwhile comparing to the simplicity and accuracy it provides in converting between SPM and PM. It is also noted that $\varepsilon_{\max } T_{0}=\varepsilon_{\max } \pi / \omega_{\mathrm{cg}, \text { nom }}$ indicates how much transport delay the nominal system can accommodates before onset of instability, which is commonly known as the delay margin, which has been proposed in the literature as a candidate for stability metrics for NL systems. However, a practical method for assessing the exact delay margin has been lacking. The method developed here provides an analytical means to evaluate the (almost) exact delay margin for a given nominal system.

\section{SUMMARY AND CONCLUSIONS}

\section{A. Summary}

The main results of this paper can be summarized as follows.

1) : Theorem 1 establishes a bijective relationship between the Singular Perturbation Margin (SPM) $\varepsilon_{\max }$ and the phase margin (PM) of the nominal system with an approximation error on the order of $\varepsilon^{2}$ for monotonic-lag fast systems. In particular, the error will be zero if the gain of the fast system is identically unity, independent of $\varepsilon$.

2) : Lemma 1 reveals the relationships between the singular perturbation parameter $\varepsilon$, PM of the perturbed system, SPM and PM of the nominal system, and the phase of the fast system at gain crossover frequency of the nominal system, which is an important result in its own right. 
3) : As an application of Theorem 1, a singular perturbation approach for time-domain PM assessment is proposed and illustrated by examples. The examples include design of PM-gauges and determination of SPM in the time domain from the state equation of the perturbed system consisting of the nominal and the PM-gauge systems. The effectiveness of this method is demonstrated with a zero-error PM-gauge design.

\section{B. Remarks}

1) : Relationship between SPM and PM of nominal system

Theorem 1 establishes a bijective relationship between the SPM and the PM of the nominal system, with an approximation error on the order of $\varepsilon^{2}$, using a monotoniclag fast system. This relation not only makes it possible to assess the phase margin of the nominal system in the time-domain for LTI systems directly using a singular perturbation approach, but most importantly it establishes a basis for using SPM as a theoretically based, practically measurable and backward compatible metric for stability margins of LTV systems and NLTV systems.

\section{2) : PM estimation error analysis}

The PM estimation error is $O\left(\varepsilon^{2}\right)$. Thus the error increases as $\varepsilon$ increases. When the nominal system has a small PM, as shown Example 1 where $P M_{\text {nom }}$ is about $20^{\circ}$, the PM-gauge offers a result with very small error. When the nominal system has a larger PM, the fast system will need a larger $\varepsilon_{\max }$ for its phase to reach the phase margin; therefore, the error will increase, as demonstrated with example 2. The $O\left(\varepsilon^{2}\right)$ PM estimation error is due to the non-unity gain $M_{\text {fast }}\left(\omega_{\mathrm{cg}, \text { nom }}\right)$ of the fast (PM-gauge) system at $\omega_{\text {cg,nom }}$, which causes the $\omega_{\text {cg,pt }}$ for the perturbed system to deviate from $\omega_{\mathrm{cg}, \mathrm{nom}}$. Thus the error can be reduced by using PM-gauge with unity gain for all $\omega$ (allpass filter) that is independent of $\varepsilon$.

\section{3) : Assessment of SPM}

The first part of Section III offers a method to determine exact SPM in the time-domain from the state equation for LTI systems. For LTV and NL systems, general methods for determining exact SPM is unlikely to exist; therefore effective estimation methods should be developed in order to use SPM as a practical stability metric. For LTV systems, a recently developed differential algebraic spectral theory [17]-[22] could be used to assess the SPM in a manner similar to the aforementioned Chang transformation and multi-time-scale decomposition for LTI systems. The LTV SPM methods, once developed, could be extended to NLTV systems by virtue of the equivalence of exponential stability of an equilibrium state of a NL system and its linearization thereof. For NL system (including LTV systems as a special class), well-known Lyapunov function based singular perturbation results (see, for instance, Theorems 11.3 and 11.4 in [11]) can be used to obtain conservative estimate of SPM. In this latter approach, a recent result on spectral Lyapunov function [23] would prove to be useful.

\section{REFERENCES}

[1] M. Dmitriev and G. Kurina, Singular Perturbations in Control Problems, Automation and Remote Control, vol.67, no.1, Jan.2006, pp.1-43.

[2] M. K. Kadalbajoo and K. C. Patidar, Singularly Perturbed Problems in Partial Differential Equations: A Survey, Applied Mathematics and Computation, vol.134, no.2/3, Jan.2003, pp.371-429.

[3] P. V. Kokotovic, R. E. O’Malley, Jr. and P. Sannuti, Singular Perturbations and Order Reduction in Control Theory: An Overview. Automatica, 12:123-132, 1976.

[4] A. Weinmann, Stability Margin and Spherical Uncertainty, Cybernetics and Systems, vol.37, no.7, Oct.2006, pp.685-705.

[5] M. Bozorg and E. J. Davison, Control of Time Delay Processes with Uncertain Delays: Time Delay Stability Margins, Journal of Process Control, vol.16, no.4, Apr.2006, pp.403-408.

[6] C. Cao, and N. Hovakimyan, "Stability Margins of L1 Adaptive Controller: Part II", Proceedings of American Control Conference, 2007, pp.3931-3936.

[7] S. A. Hodel, M. Whorton, J. J. Zhu, "Stability Metrics for Simulation and Flight-Software Assessment and Monitoring of Adaptive Control Assist Compensators", AIAA-2008-7005, Proceedings, AIAA Guidance, Navigation and Control Conference, Honolulu, HI, August, 2008.

[8] C. K. Pang, F. L. Lewis, S. S. Ge, G. Guo, B. M. Chen and T. H. Lee, Singular Perturbation Control for Vibration Rejection in HDDs Using the PZT Active Suspension as Fast Subsystem Observer, IEEE Trans. on Industrial Electronics, vol.54, no.3, June 2007, pp.1375-1386.

[9] A. Saberi and H. K. Khalil, Quadratic-type Lyapunov functions for singularly perturbed systems, IEEE Trans. on Automatic Control, vol.29, no.6, 1984, pp.542-550.

[10] L. K. Kuzmina, Some Problems in Stability Theory of Singularly Perturbed Systems with Multiple Time-scales, Nonlinear Analysis, vol.63, 2005, pp.1289-1297.

[11] H. K. Khalil, Nonlinear Systems, Prentice Hall, Upper Saddle River, NJ; 2002.

[12] P. V. Kokotovic and A. H. Haddad, Controllability and Time-Optimal Control of Systems with Slow and Fast Modes, IEEE Trans. on Automatic Control, vol.20, no.1, 1975, pp.111-113.

[13] P. V. Kokotovic, H. K. Khalil, and J. O'Reilly, Singular Perturbations Methods in Control: Analysis and Design, New York: Academic Press, 1986.

[14] K. W. Chang, Singular Perturbations of A General Boundary Value Problem, SIAM J. Math. Anal., vol.3, 1972, pp.520-526

[15] N. Prljaca and Z. Gajic, General Transformation for Block Diagonalization of Multitime-Scale Singularly Perturbed Linear Systems, IEEE Trans. on Automatic Control, vol.53, no.5, 2008, pp.1303-1305.

[16] G. A. Kurina, Complete Controllability of Singularly Perturbed Systems with Slow and Fast Modes, Mathematical Notes, vol.52, no.4, 1992 pp.1029-1033.

[17] F. Colonius and W. Kliemann, The Lyapunov Spectrum of Families of Time-Varying Matrices, Transactions of American Math. Society, vol.348, no.11, 1996, pp.4389-4408.

[18] J. J. Zhu, A Note on Extension of the Eigenvalue Concept, IEEE Control Systems Magazine, vol.13, Dec.1993, pp.68-70.

[19] J. J. Zhu, C. D. Johnson, New Results in the Reduction of Linear Time-Varying Dynamical Systems, Society for Industrial and Applied Mathematics, vol.27, no.3, May 1989, pp.476-494.

[20] J. J. Zhu, "A Necessary and Sufficient Stability Criterion for Linear Time-Varying Systems", Proceeding, 28th IEEE Southeastern Symposium on Systems Theory, Baton Rouge, LA, April 1996, pp.115-119.

[21] J. J. Zhu, "PD-eigenstructure Assignment Control for Multivariable Nonlinear Tracking and Decoupling", Final Report, AFOSR Summer Research Extension Program, Dec.1996.

[22] P. J. Antsaklis, A. N. Michel, Linear Systems, McGraw-Hill Companies, INC., Chap.3, 1997.

[23] J. J. Zhu, Y.Liu and R.Hang, "A Spectral Lyapunov Function for Exponentially Stable LTV Systems", Proceedings, 2009 American Control Conference, June 2009. 